\title{
La Mandragore ou Le Bourgeois de Szymanowski
}

Didier van Moere

\section{OpenEdition}

Journals

Édition électronique

URL : http://journals.openedition.org/recherchestravaux/286

DOI : 10.4000/recherchestravaux.286

ISSN : 1969-6434

\section{Éditeur}

UGA Éditions/Université Grenoble Alpes

\section{Édition imprimée}

Date de publication : 30 mai 2005

Pagination : 211-219

ISBN : 0151-1874

ISSN : 0151-1874

\section{Référence électronique}

Didier van Moere, "La Mandragore ou Le Bourgeois de Szymanowski », Recherches \& Travaux [En ligne], 67 | 2005, mis en ligne le 27 janvier 2014, consulté le 08 septembre 2020. URL : http://

journals.openedition.org/recherchestravaux/286 ; DOI : https://doi.org/10.4000/recherchestravaux 286 


\section{La Mandragore ou Le Bourgeois de Szymanowski}

À l'heure où la mode est plus aux reconstitutions qu'aux adaptations, nul metteur en scène ne songerait sans doute à remplacer, dans Le Bourgeois gentilhomme de Molière, le Ballet des nations par un divertissement de son invention ${ }^{\mathrm{I}}$. Certes rien n'empêche de penser que Strauss et Hofmannsthal écriraient aujourd'hui la seconde version d'Ariane à Naxos, totalement affranchie du Bourgeois grâce au prologue, où «l'homme le plus riche de Vienne» remplace Monsieur Jourdain. Il est douteux, en revanche, que la première version, qui ramène à deux actes la comédie de Molière $^{2}$ et où Monsieur Jourdain offre comme divertissement à ses invités l'opéra mythologique, soit encore pensable. Peut-être en est-il de même du Bourgeois Gentilhomme 3 créé à Varsovie le Is juin I920 au Teatr Polski (Théâtre polonais), sous la forme d'une "comédie en trois actes agrémentée d'un ballet", en l'occurrence La Mandragore de Karol Szymanowski, sur un argument de Ryszard Boleslawski ${ }^{4}$ et Leon Schiller ${ }^{5}$. Si ces derniers, en effet, sont incontestablement plus fidèles à

I. Récemment, V. Dumestre et son Poème harmonique ont proposé une reconstitution du Bourgeois gentilhomme tel qu'il fut donné en 1670 au château de Chambord, dans une mise en scène de B. Lazar. Sur cette première version, voir l'édition de J. Serroy, Paris, Gallimard (Folio), p. 257-265.

2. «[... ] je supprime la cérémonie turque [...] elle est intraduisible [...] toute l'action parallèle, avec les personnages de la fille, de Cléante, de Covielle, c'est-à-dire un tiers de la pièce, devient inutile.» Lettre du I5 mai I9II de Hofmannsthal à Strauss, dans Correspondance 1900-1929, trad. B. Banoun, Paris, Fayard, 1992, p. I20.

3. La traduction était due au célèbre traducteur T. Boy-Zelenski.

4. R. Boleslawski (1889-1937): acteur, metteur en scène de théâtre et de cinéma.

5. Metteur en scène, critique, historien du théâtre, chansonnier et compositeur, Leon Schiller (I887-I954) avait, après avoir étudié à Cracovie, Paris et Vienne, débuté en publiant des essais dans la revue de E. G. Craig Le Masque en 1908. Il fut directeur littéraire et musical du Teatr Polski de I9I7 à I92I. Sa collaboration avec Szymanowski ne se limita pas à La Mandragore: il fit appel à lui pour la musique de scène du dernier acte du Prince Potemkine, un drame de T. Micinski, créé le 6 mars I925 à Varsovie; il assura ensuite, en collaboration avec S. Lifar, la mise en scène à l'Opéra de Paris du ballet Harnasie, dont la première eut lieu le 27 avril 1936. 
Molière $^{6}$, leur adaptation n'en posait pas moins la question de la musique dans les pièces du répertoire ancien.

Schiller l'avait résolue à sa manière:

Un type tout à fait nouveau d'illustration musicale vit le jour au Teatr Polski à l'occasion de représentations d'œuvres comme Monsieur de Pourceangnac ou Le Bourgeois gentilhomme de Molière et Le Barbier de Séville de Beaumarchais. La méthode employée par Leon Schiller consistait à associer diverses compositions de l'époque d'un auteur donné à ses propres œuvres, qui étaient des stylisations à l'ancienne. Ainsi, dans Monsieur de Pourceangnac, Schiller, pour les passages dansés ou les entractes, utilisa des compositions de Lully ainsi que de ses contemporains français ou italiens, tandis qu'il écrivit lui-même tous les solos et les chœurs, ainsi que les entrées, s'adaptant aux indications de mise en scène, veillant exactement lors de toutes les répétitions à la concordance des effets musicaux avec le jeu des acteurs et le ton d'ensemble de la mise en scène. [...] Il s'en tint au même principe $[\ldots]$ pour la partie musicale du Barbier de Séville. Il choisit des passages musicaux dans les opéras et ballets de l'époque, tandis qu'il composa les parties chantées dans l'esprit et la manière de Monsigny, Martini, Rousseau et Mozart.7

S'agissant du Bourgeois, l'affiche et le programme indiquent que «la partie musicale de la comédie de Molière a été révisée et complétée par Leon Schiller d'après des motifs de Lully». Concrètement, n'avaient été retenus que l'ouverture, le célèbre menuet et trois courts passages de la cérémonie turque; tout le reste était de son cru. Trouvant la musique élégante de Lully, malgré son charme incontestable, trop indissolublement liée au théâtre de cour, Schiller voulait une illustration plus vivante, plus piquante, plus colorée, qui ne trahît pas pour autant l'esprit de son prédécesseur ${ }^{8}$. Restait à convaincre Szymanowski de se lancer dans l'aventure. Or ce dernier n'aime guère la contrainte des commandes et travaille parfois lentement. Il est pourtant aussitôt séduit et imagine le canevas de l'histoire, avec Schiller et Boleslawski, dans le petit jardin du théâtre. Schiller souhaite un divertissement dans l'esprit de la commedia dell'arte, dont on doit retrouver des personnages traditionnels. Pour bien le distinguer des parties dansées, on en fera une pantomime, confiée à des acteurs, qui restera étroitement liée à l'action de la comédie par l'intervention, à la fin, de Monsieur Jourdain, sur une partie du menuet de Lully. Schiller met la dernière main au scénario le soir même et le

6. L'ensemble des personnages est conservé, de même que la cérémonie turque.

7. H. Opieński, cité par T. Chylińska dans K. Szymanowski, Correspondance générale, t. II (I920-I926) vol. I, Cracovie, Éditions polonaises de musique, I994, p. 96-97. Musicologue, compositeur, chef d'orchestre et critique, Opieński (1870-1942) avait été le prédécesseur de Schiller au Teatr Polski.

8. À en croire Opieński, il y parvint si bien que plus d'un critique ou d'un musicologue, même parmi les plus avertis, n'y virent apparemment que du feu. 
transmet à Szymanowski. Ce dernier, chaque jour, lui donne ce qu'il a composé et termine La Mandragore en un peu plus d'une semaine.

Destinée à un petit orchestre 9 , d'une durée d'à peine vingt minutes, la pantomime se compose de trois tableaux. À Houhoubambou, le Roi Sinadab s'ennuie, irrité des cajoleries de la Reine Gulinda dont il est las et qui essaie vainement de raviver son désir par une danse du ventre. L'Eunuque introduit alors Colombine voilée; quand il voit son visage, le Roi est transporté, à la grande fureur de la Reine qui bourre sa rivale de coups de poing; pour s'en débarrasser, le royal époux la fait enfermer dans la cage du perroquet. À la demande du Roi, Colombine se livre à "une danse lente, pleine de nostalgie et de tristesse». On entend alors, en coulisse, une aria langoureuse : c'est Arlequin. Colombine se remet à danser, mais cette fois avec une sensualité charmeuse, en profitant pour enlever au Roi ses bijoux. Ce dernier l'entrâne dans ses appartements, alors qu'on entend à nouveau Arlequin et que l'Eunuque délivre la Reine. Au deuxième tableau, la tempête fait rage: Arlequin, le docteur bolonais et le capitaine Cocodrillo échappent de justesse à la noyade; le Docteur les ramène à la vie avec un clystère. Arlequin, désespéré, couvre de baisers le portrait de Colombine et supplie le Capitaine de la délivrer. C'est justement ce qu'elle demande désespérément quand elle apparaît à la fenêtre. Le matamore se précipite, sabre au clair, mais il s'évanouit à la vue du roi qui les nargue: le docteur, une fois de plus, utilise son clystère. La Reine arrive alors de la coulisse et donne une grande clé à Arlequin. Le Capitaine s'évanouit de nouveau, toujours soigné par le même remède. Le tableau s'achève sur une danse de joie. Au dernier tableau, nous retrouvons le Roi somnolant, "épuisé par la passion ", éventé par Colombine qui lui chatouille le nez et les talons avec une plume. Les trois compères arrivent, le fanfaron se précipite sous une table pour se cacher. Colombine tombe dans les bras d'Arlequin, tandis que le Docteur administre au Roi de la mandragore. Mais l'effet en est bref: le Roi se réveille et poursuit Arlequin. Monsieur Jourdain arrive à point pour les séparer, sur la musique du menuet de Lully: le Roi le regarde interloqué. Le Bourgeois gentilhomme lui amène alors deux femmes voilées: croyant choisir Colombine, le Roi choisit la Reine, à la grande joie des autres. Il s'évanouit, la mandragore le ranime et il se lance dans une «danse sauvage», bientôt rejoint par la Reine. Les autres dansent de leur côté, le clystère du Docteur ranimant une dernière fois le Capitaine. Monsieur Jourdain tire enfin les rideaux de l'alcôve pour que le public ne voie pas le couple royal.

9. Flûte (et piccolo), hautbois, clarinette en do, hautbois, deux cors en fa, timbales, triangle, cymbales, petit tambour, tambour de basque, piano, trois premiers violons, deux seconds violons, un alto, un violoncelle et une contrebasse. 
L'Eunuque écoute un moment derrière le rideau, "rempli de dégoût et de mépris» pour ce qu'il entend; il disparaît ensuite dans la coulisse, "fredonnant une chanson triste à mi-voix", le perroquet sur son épaule.

L'histoire, on le voit, est comique et impose à la musique de faire rire aussi, d'autant plus que Szymanowski a suivi scrupuleusement les indications de Schiller. Le comique musical passe d'abord par des effets de pure imitation, consacrés par l'usage. Les bâillements du Roi sont accompagnés, au début, par des portamenti des violoncelles jouant sul una corda la quinte si bémol-fa $(\mathrm{p} .4-5)^{\mathrm{IO}}$, tandis que le basson - souvent associé, en musique, à l'ironie - fait entendre une phrase piquante. Lorsque commence le troisième tableau, des glissandi, cette fois étendus à toutes les cordes, soulignent la somnolence du Roi (p. 7I-74). Pour la tempête, Szymanowski a recours à tous les procédés traditionnels: roulements de timbales - doublés par les tremolos du piano, grands arpèges ascendants et descendants des altos et des violoncelles, fusées chromatiques descendantes des premiers violons, tremolo des seconds violons et des contrebasses, sifflements stridents du piccolo, avant que des contretemps ne disloquent le rythme (p. 42-44). Larrivée du Capitaine est tout aussi caractéristique: arpège descendant de septième diminuée joué pizzicato par le violoncelle, doublé par les notes pointées du basson pour son évanouissement, glissando descendant de trois octaves au piano pour l'administration du clystère, arpège ascendant d'accord parfait majeur au cor sur roulement de timbale pour la fanfare soulignant son réveil en trombe; lorsqu'il s'évanouit à nouveau, à la vue du Roi, les cordes jouent tremulando sulponticello une descente chromatique de quatre notes conjointes (p. 46-47).

Il arrive aussi que la musique soit ouvertement parodique. La parodie peut être explicitée par les indications portées sur la partition. L'évocation de Colombine par l'Eunuque, au premier tableau, faite «avec emphase et exagération", trouve son écho dans une phrase du hautbois jouée con una espressione parodistica (p. I6); la même indication accompagne une autre phrase du hautbois, toujours associé à l'Eunuque, dont il souligne, à la fin, les gestes de mépris pour les ébats du couple royal (p. I05-IO6). La parodie peut également relever du clin d'œil amusé et viser un genre. L'air d'Arlequin vient tout droit de ces opéras italiens où le ténor transi attend que sa belle lui accorde ses faveurs, se promettant de ne pas survivre à un refus, terminant par une vocalise flatteuse pour la voix (p. 27-29, p. 37-38). L'ombre de Bellini et de

IO. Nous nous référerons à la partition publiée en 1949 aux Éditions polonaises de musique (Cracovie), dans le cadre de l'intégrale des œuvres de Szymanowski, dont elle constitue le tome 25. On notera que le fa est appoggiaturé, comme le si bémol tenu des contrebasses, ou certaines notes de la flûte et du hautbois. 
Donizetti ${ }^{I I}$, que Szymanowski n'aimait guère, plane de toute évidence sur cette sérénade accompagnée par le seul piano, qui doit ici imiter la harpe ${ }^{\mathrm{I2}}$. Mais la parodie, dans certains cas, est beaucoup plus implicite; sans qu'on tombe dans le pastiche, qui est une imitation clairement revendiquée, le sourire s'y mêle à l'hommage. Ainsi, dans le troisième tableau, au menuet de Lully - où Monsieur Jourdain "se trompe un peu dans le rythme" - succède une valse lente, sur laquelle danseront les «filous» tout heureux du succès de leur ruse, dont on peut se demander si elle ne serait pas un clin d'œil adressé à Richard Strauss ${ }^{13}$. Or Szymanowski a éprouvé pour Strauss, le premier à lui avoir révélé la modernité, une admiration éperdue, avant de s'en détourner pour adopter l'esprit de l'impressionnisme musical. À l'époque de La Mandragore, il ne risque donc plus de succomber de nouveau à cette «straussomanie» dont il a lui-même, à propos de l'opéra Hagith, mesuré le danger ${ }^{\mathrm{I4}}$. Finalement, la parodie relève ici de l'emprunt d'un procédé dont on a éprouvé la pertinence chez un modèle à qui on témoigne du même coup sa reconnaissance; cet emprunt, dans le passé, aurait pu signifier une allégeance et relever du pur mimétisme; il n'est ici rien de plus qu'un hommage, alors que, dans le cas de l'air d'Arlequin, il tient presque de la charge, ne serait-ce que parce que l'opéra italien, loin d'être ou d'avoir été un modèle à imiter, est plutôt un exemple à ne pas suivre. Strauss lui-même, dans son Ariane à Naxos, n'avait-il pas associé le rythme de la valse aux personnages de la commedia dell'arte? Le début de la danse sauvage du couple royal, en revanche, avec ses accents sur le temps faible, rappelle plutôt Stravinski, celui de Petrouchka et du Sacre du printemps ${ }^{15}$, de même que les effets de contretemps épousant, au premier tableau, les sauts grotesques du Roi affolé par Colombine dans un passage où les «fausses notes» du basson et de l'alto prêtent également à sourire (p. 3536). Le comique, selon un procédé là aussi éprouvé, vient de la dissonance

II. Vue à New York en janvier I92I, Norma l'ennuie. Sur Verdi, l'opinion de Szymanowski évoluera et il déclarera «beaucoup apprécier» dans une interview donnée en novembre 1933 (voir Écrits musicaux, Cracovie, Éditions polonaises de musique, 1984, p. 436).

I2. Pour bien nous inviter à ne pas être dupes, Szymanowski, dans la version de concert, remplace le ténor par le basson. Szymanowski a-t-il pensé au ténor italien du Chevalier à la rose de Strauss, qu’il avait vu au moins deux fois à Vienne en juin I9II? Ce n'est pas si sûr: Strauss a en réalité écrit une musique très ambiguë, qui ne se réfere pas à Bellini ou à Donizetti et dont le caractère parodique, malgré les apparences, reste peut-être à démontrer.

I3. Pas seulement au Chevalier à la rose, où la valse est omniprésente. Il y a aussi des valses, ou du moins des rythmes de valse, dans des œuvres de Strauss qui sont rien moins que comiques, d'Ainsi parlait Zarathoustra à Elektra, en passant par Salomé (op. cit., p. 86-90).

I4. Lettre du 24 septembre I9ı2 à Zdzislaw Jachimecki, Correspondance générale, t. I, p. 356. I5. Si Szymanowski a entendu Petrouchka à Vienne dès 19I2, il n'entendra Le Sacre qu'en I92I à Paris. Mais il connaissait l'œuvre, qu'il jouait au piano dès I9I3, l'année de sa création mouvementée à Paris (op. cit., p. 95-98). 
provoquée par l'intrusion de notes étrangères. Ainsi en est-il par exemple des accords appoggiaturés entendus lorsque le Roi, au premier tableau, se montre à la fenêtre ou, au troisième, se réveille, provoquant une même panique ${ }^{16}$. La parodie fonctionne ici de la même manière, ce qui mérite d'autant plus d'être souligné que Szymanowski éprouve toujours la même fascination pour Stravinski, qui justement l'a aidé à se guérir de Strauss ${ }^{17}$.

Mais que dire des passages où Szymanowski semble se pasticher lui-même? Certains effets sonores, notamment ceux qu'il tire des cordes, sont empruntés aux œuvres de sa période «impressionniste», à laquelle il vient pourtant de mettre un terme en s'engageant, au moment où la Pologne vient de recouvrer son indépendance, dans le combat de la musique nationale: effets de glissando ou de tremolo, jeu sul ponticello, etc. L'Andante languido de la musique de Colombine, au premier tableau, renoue avec la sensualité de la Troisième Symphonie ou du Premier Concerto pour violon: chant éperdu des deux premiers violons dans l'aigu accompagnés par le reste des cordes divisé, deux des seconds violons jouant pizzicato quasi chitarra, légère touche d'exotisme introduite à la fois par certains intervalles et par le tambour de basque qui souligne le rythme de la danse (p. 25-27). La reprise de la danse dans l'allegretto grazioso est tout aussi éloquente, avec ses arabesques de la flûte, ses effets de polyrythmie, le saltando d'une partie des cordes, le premier violon au premier plan toujours dans l'aigu, voire le suraigu, la partie de piano surtout, qui est traité comme l'égal des autres instruments (p. 3I-34). On ne dira évidemment pas que l'emprunt relève ici de l'hommage narcissique. Il pourrait bien, en revanche, exprimer une certaine nostalgie, pourtant sans illusion, de cette période impressionniste dont les œuvres avaient été nourries de ses éblouissements méditerranéens, qui connaitront un dernier accomplissement dans ce Roi Roger que Szymanowski est en train d'esquisser alors que l'idée lui en est venue en $1918^{18}$. On peut d'ailleurs voir dans $L a$ Mandragore une sorte de double comique, grotesque même du Roi Roger. À l'Orient capiteux de l'opéra répond l'Orient de pacotille de la pantomime. À la transe dionysiaque de Roxane habillée en Ménade fait écho la danse sauvage du couple royal - où la

I6. Référence au troisième tableau de Petrouchka, où les accords appogiaturés abondent? (op. cit., p.57, 83).

17. Si Szymanowski a rencontré Stravinski à Londres en I9I4. Au retour de son voyage à Paris en I92I, Szymanowski écrira un article enthousiaste sur le compositeur russe et composera ses Słopiewnie (Archaïques), où l'on sent l'ombre de Stravinski. Ce dernier sera encore présent dans le ballet Harnasie (Les Brigands), achevé en 1931, que Roland-Manuel appellera ironiquement, au moment des représentations parisiennes de 1936, "Le Sucre du printemps» (Le Courrier royal, 6 mai 1936).

I8. Il ne sera achevé qu’en 1924. Sur le Roi Roger, voir notre présentation de la version de concert donné au Châtelet les 25 et 27 avril 2003. 
Reine agite un tambourin pour marquer le rythme de la danse comme Roxane agite un thyrse. Et tout commence, dans les deux cas, par la décomposition d'un couple royal, qu'aggrave encore l'arrivée d'un personnage qui va exercer sur le souverain une étrange fascination - le Berger d'un côté, Colombine de l'autre.

Peu analysée par les spécialistes de Szymanowski, La Mandragore est sans doute plus que cette simple " arlequinade $^{19}$ " dont il parlait lui-même avec une certaine condescendance. Certes il songe immédiatement à «l'étoffer un peu et [à] la séparer tout à fait du Bourgeois, pour qu'elle puisse être exécutée de façon autonome "(ibid.). Il l'emporte d'ailleurs avec lui aux États-Unis, où cette version de concert est créée le 20 janvier 1922 à New York ${ }^{20}$. La perspective d'exécutions en Europe suscite en revanche chez lui de grandes réserves. Il cède à Albert Coates qui, après l'avoir entendue à New York, tient absolument à la diriger à Londres, alors que Szymanowski craint qu' on ne la «prenne au sérieux ${ }^{2 \mathrm{II}}$. Mais il refuse catégoriquement qu’on la donne à Paris: «[...] la petite musique pour orchestre de chambre était assez bonne pour ce grand imbécile de New York [...] mais pas assez bonne pour Paris ${ }^{22}$.»

La Mandragore est pourtant dans l'esprit du temps, où la commedia dell'arte inspire les compositeurs. L'Arlecchino de Busoni a été créé à Zurich en I9I7, l'Amour des trois oranges de Prokofiev va l'être à Chicago en I92I ${ }^{23}$. Mais c'est surtout avec Pulcinella de Stravinski, créé à Paris juste un mois avant $\mathrm{La}$ Mandragore, qui est aussi un ballet empruntant à la commedia dell'arte, qu'il faut comparer la pantomime de Szymanowski. Malgré les apparences, tout sépare ici les deux musiciens. Stravinski compose Pulcinella "d'après Pergolèse ", adoptant sans ambages une perspective néoclassique relevant du pastiche que l'on retrouvera jusque dans The Rake's Progress de 1951, quitte à épicer parfois la musique de savoureuses dissonances ou à ajouter à l'orchestre un instrument aussi anachronique que le trombone ${ }^{24}$ alors que, par souci

I9. Lettre (en allemand) du 23 juin 1923 à E. Hertzka, Correspondance générale, op. cit., t. II, vol. 3, p. I69.

20. La Mandragore sera donnée sous sa forme chorégraphique en novembre 1925 à Chicago, comme ouverture de la saison du Ballet intime de A. Bolm, un ancien des Ballets russes.

2I. Lettre (en français) postérieure au I7 juin I922 à M. Coates, Correspondance générale, t. II, vol. 3, p. 77 .

22. Lettre (en français) du 22 novembre I922 à H. Kahn-Casella, ibid., p. IO2 (je souligne).

23. En février 1922, Szymanowski assistera à une des représentations données à New York par l'Opéra de Chicago en tournée.

24. Ses glissandi, dans le Vivo, associés à la contrebasse, auraient du mal à rappeler Pergolèse! 
d'authenticité, il a renoncé à la clarinette ${ }^{25}$. Le néoclassicisme ne tentera jamais Szymanowski: "Karol est avec Ravel le seul compositeur qui n'ait pas sacrifié aux retours ${ }^{26}$." La Mandragore reste une parenthèse dans son œuvre alors que Pulcinella correspond à un renouvellement profond du langage de Stravinski. Alors que Szymanowski devient un compositeur polonais et rentre dans sa patrie, Stravinski devient un exilé et cesse d'être un compositeur russe $^{27}$. Le rapport entre la scène et la fosse est également conçu de façon tout à fait différente: si la musique de Szymanowski, on l'a vu, suit l'action au plus près, celle de Stravinski maintient résolument une distance:

Pulcinella est plutôt une "action dansante» qu'un ballet [...]. Comme dans Les Noces, les chanteurs [qui sont dans la fosse] ne s'identifient pas aux personnages évoluant sur la scène. Ils chantent "dans le caractère» de ce qui s’y passe - sérénades, duos, trios - de véritables numéros interpolés. ${ }^{28}$

Même si La Mandragore constitue un hapax dans l'œuvre de Szymanowski, ce dernier n'a pas dû, pour l'écrire, forcer sa nature. Elle renoue avec ces divertissements facétieux qu'il organisait avec ses sœurs, son frère et ses cousins du temps de sa jeunesse insouciante, à Tymoszowka ou à Elisavetgrad. Le créateur du rôle du Docteur est d'ailleurs son cousin Feliks Zbyszewski, qui faisait partie de la troupe du Teatr Polski ${ }^{29}$. Lui-même avait, en I909, composé une opérette, La Loterie aux maris, se réclamant à la fois de l'opérette viennoise et de la comédie musicale américaine ${ }^{30}$. Mais ses œuvres les plus sérieuses, en particulier celles pour piano, témoignent très tôt, ici ou là, d'un certain goût pour la parodie. Faut-il prendre au sérieux, dans les Variations $o p$. 3, la valse de la neuvième variation? le Menuet de la Première Sonate? celui des variations de la Deuxième - joué pomposo? L'invitation à la danse qu'adresse l'Ermite défroqué à des jeunes filles, dans la première mélodie de l'opus $I 7$, avec ses accents sur les temps forts ou les demi-temps, ses syncopes, ses effets de martèlement suggérant le piétinement de l'ermite en train de danser,

25. Toute percussion a aussi disparu de l'orchestre de Pulcinella, où Stravinski revient à l'opposition entre le concertino et le ripieno.

26. Lettre du 26 janvier 1932 de H. Kahn-Casella à J. Iwaszkiewicz, Bibliothèque universitaire de Varsovie, Archives des compositeurs polonais. Le cas de Ravel est cependant plus complexe qu'il n'y parait.

27. Noces a été créé en 1923, mais commencé en I9I4. Quant au Baiser de la fée de 1928, il est - encore - un hommage-pastiche à Tchaikovski, le plus occidentalisé des musiciens russes, sur un argument tiré de La Vierge des glaciers d'Andersen.

28. Notice de l'enregistrement Sony, publié dans le cadre de la quasi-intégrale réalisée sous la direction du compositeur.

29. C'est peut-être lui qui suggéra à Leon Schiller de solliciter Szymanowski.

30. Il y voyait surtout un moyen de se faire un nom et de gagner de l'argent; prudemment, il la signa du nom d'un pseudonyme, "Withney». 
prêtent à sourire, comme prêteront à sourire beaucoup des Enfantines ${ }^{3 \mathrm{I}}-$ ou la piquante Valse romantique. Pour ne rien dire de l'ironie mordante que Szymanowski a mise dans les fugues finales de la Deuxième et de la Troisième Sonate, où la musique bouffonne dès l'énoncé des sujets, tout à sauts et à gambades - poco scherzando e capriccioso pour la Deuxième, scherzando e buffo pour la Troisième. Ou de la polytonalité du scherzo du Premier Quatuor ${ }^{32}$. La Mandragore, en revanche, échappe aux grimaces et aux rictus défigurant, dans Masques, Tristan ou don Juan, qui sont en réalité des figures tragiques ${ }^{33}$.

Les trente et une représentations du Bourgeois gentilhomme et de La Mandragore remportèrent un grand succès auprès d'un public parfois surpris par la musique de Szymanowski. La critique se montra plutôt favorable également, même si certains apprécièrent peu le mélange des genres, trouvant qu'il nuisait à l'œuvre de Molière. Ainsi Stanisław Pieńkowski fait-il l'éloge de la pantomime et de la musique de Szymanowski, mais déplore qu'elles aient été associées au Bourgeois: "On s'est presque conduit avec [Molière] comme le tailleur avec Monsieur Jourdain ${ }^{34}$." Stanisław Niewiadomski, qui n’a jamais été très favorable à Szymanowski, pense de même et aurait préféré aussi qu'on gardât la musique de Lully même s'il reconnaît des qualités à celle de la pantomime, qui devrait finalement être déconnectée de la comédie de Molière: «[...] Szymanowski, malgré toutes les concessions faites au public dans le domaine de la mélodie, n’a pu se défaire de son style dissonant 35 ». On en revenait, une fois de plus, à l'éternelle question de l'authenticité.

3I. Que dire, par exemple, de la quatrième, où le goret se pavane sur un rythme de mazurka? 32. Il est écrit en quatre tonalités, constituant une septième diminuée (do majeur au violoncelle $-m i$ bémol à l'alto $-f a$ dièse majeur et $l a$ majeur aux violons). Le finale n'ayant jamais été écrit, ce scherzo en tient lieu.

33. "Tantris le bouffon" et la "Sérénade de don Juan" sont les deuxième et troisième Masques.

34. Le Journal de Varsovie, I9 juin 1920.

35. La Gazette musicale, juillet 1920. 